\title{
Essais
}

Revue interdisciplinaire d'Humanités

$10 \mid 2016$

Faire-valoir et seconds couteaux

\section{Fulvio Cammarano, Abbasso la guerra! Neutralisti in piazza alla vigilia della prima guerra mondiale in Italia}

\section{Eleonora Lega}

\section{(2) OpenEdition}

Journals

Édition électronique

URL : http://journals.openedition.org/essais/4203

DOI : $10.4000 /$ essais.4203

ISSN : 2276-0970

Éditeur

École doctorale Montaigne Humanités

Édition imprimée

Date de publication : 15 septembre 2016

Pagination : 185-187

ISBN : 978-2-9544269-9-0

ISSN : 2417-4211

Référence électronique

Eleonora Lega, « Fulvio Cammarano, Abbasso la guerra! Neutralisti in piazza alla vigilia della prima guerra mondiale in Italia », Essais [En ligne], 10 | 2016, mis en ligne le 15 octobre 2020, consulté le 25 octobre 2020. URL : http://journals.openedition.org/essais/4203; DOI : https://doi.org/10.4000/essais.4203 


\section{Comptes rendus}

F. Cammarano, Abbasso la guerra! Neutralisti in piazza alla vigilia della prima guerra mondiale in Italia, Mondadori, Milano, 2015.

A. Lepre, C. Petraccone, Storia d'Italia dall'Unità ad oggi, Mulino, Bologna, 2003, p. 123-150.

L'historiographie italienne s'étant souvent penchée sur la thématique de l'avant première guerre mondiale, on s'attendrait à un grand nombre d'études sur le mouvement pacifiste. Il faut ajouter également que même les contemporains avaient conscience de la singularité de l'Italie qui, contrairement aux autres puissances européennes, a pu garder une position de neutralité pendant neuf mois, de juillet 1914 à mai 1915. Pourtant, avant la publication de cette œuvre, le mouvement de masse pacifiste a toujours été un objet d'études marginal. Fulvio Cammarano, professeur d'histoire contemporaine auprès de l'Université de Bologne et, depuis septembre 2015, président de la Société Italienne pour l'Étude de l'Histoire Contemporaine, a décidé de pallier à une lacune historiographique et de s'interroger sur la signification de ce mouvement et sur son ampleur effective. Pour ce faire il a rassemblé dans un seul ouvrage " composite et non exhaustif » cinquante essais, qui, s'appuyant sur des recherches inédites, approfondissent l'étude du mouvement neutraliste à la fois dans les villes et dans les campagnes. Publié à l'occasion du centenaire de l'entrée en guerre de l'Italie, cet ouvrage qui tâche de documenter l'histoire de tous ceux qui manifestaient dans les rues en criant " $\grave{A}$ bas la guerre!" surgit de l'exigence de s'interroger à nouveau sur la signification du mouvement neutraliste, de ses courants internes et de son ampleur. 
Il est intéressant de remarquer la structure du livre, qui est articulée en deux parties. La première est dédiée à une réflexion sur les idéologies et les pratiques différentes qui opposent les courants du mouvement neutraliste pendant ces neuf mois critiques. C'est pourtant la seconde partie qui est la plus engageante, car il s'agit de trente-huit essais qui décrivent le contexte dans lequel agissaient les mouvements neutralistes de Nord à Sud, se penchant sur les pratiques et sur les particularités locales dans le but d'en tirer des éléments de comparaison.

Dans la première partie, une série d'essais analyse les difficultés des mouvements qui se reconnaissent dans l'esprit neutraliste. En premier lieu, ils sont divisés par des différences idéologiques profondes, qui les empêchent de lutter les uns à côté des autres. C'est notamment le cas des socialistes et des catholiques, la violente critique de la presse catholique au manifeste du 21 septembre en étant d'ailleurs la preuve. En second lieu, il y a la puissance des idéaux du Risorgimento et de l'Irredentismo qui, comme le démontre l'essai sur le neutralisme anarchiste, reste séduisante même au sein du mouvement qui avait été considéré le plus uni par l'historiographie. Les catholiques ne faisaient pas exception non plus. Un essai analysant la position du Pape face au clergé relève qu'il avait dû rappeler maintes fois que le neutralisme était la voie à suivre. De plus, une fois l'Italie entrée en guerre, il avait ordonné aux curés de ne pas se laisser emporter par l'enthousiasme. Pendant les fonctions, par exemple il les avait empêché de bénir les drapeaux de l'Italie. Dans ce contexte, une véritable exception est constituée par le mouvement pour l'émancipation des femmes, qui avait déjà été mis à l'épreuve pendant les contestations à l'heure de la guerre de Lybie où les féministes avaient forgé le slogan " Ni un sou, ni un soldat ». Le mouvement des femmes demeura très actif et démontra une cohésion interne pendant les neuf mois de neutralité, même à l'heure où les affrontements entre les interventistes et les neutralistes s'aiguisaient. Dans le même essai on rappelle que, pendant cette période, une délégation internationale des femmes fut même créée à la Haye et fut reçue par les gouvernements de Londres, Berlin et Vienne. Elle ne fut pas reçue à Rome avant le mois de juin, quand l'Italie avait déjà fait son entrée en guerre. D'ailleurs, comme le démontre efficacement l'essai sur la politique étrangère italienne, le ministère des affaires étrangères suivait une realpolitik qui ne s'inspirait ni des idéaux interventistes, ni de ceux neutralistes.

Dans la seconde partie le but de dresser un bilan de l'impact du phénomène neutraliste est évident. Dans les lignes générales, les clivages retracés par l'historiographie traditionnelle entre Nord et Sud et entre ville et campagne sont confirmés. Pourtant, le travail d'archive a donné quelques résultats qui s'écartent complètement de ce modèle. Les études qui composent cette partie du livre se construisent à partir d'un corpus de sources locales, telles que les communications officielles, les ordonnances promulguées par les institutions et les publications faites par les associations présentes sur le territoire. Elles sont également basées sur les évènements parus dans la presse locale concer- 
nant les réunions publiques, les manifestations, les affrontements dans les rues, les rassemblements, les processions, les messes, les prédications.

C'est effectivement dans les grandes villes du Nord (Turin, Gênes et Milan) où les chercheurs ont retrouvé des mouvements neutralistes très forts et idéologiquement marqués. En effet, cela a même amené à des émeutes et à des affrontements violents entre neutralistes et interventistes, qui au fur et à mesure que les mois s'écoulaient, préoccupaient de plus en plus les institutions. Un témoignage de ces inquiétudes a été retrouvé dans les lettres que les préfets envoyaient à Rome où la peur de la guerre civile se répandait de plus en plus. Au contraire, la ville de Bari est un cas d'étude surprenant. Si les études sur la Calabre et sur les Abruzzes décrivent des masses lobotomisées, résignées et soumises aux pouvoirs locaux, à Bari on retrouve une forte idéologisation des masses, soutenue par les administrations locales en grande majorité socialistes. Cela se révèle une épée à double tranchant, car le mouvement nationaliste interventiste finit par l'emporter.

Plusieurs études permettent aussi de relire le mythe qui s'est crée autour des régions dites "rouges ", c'est-à-dire l'Émilie-Romagne et la Toscane. De fait, les régions où les institutions craignaient le plus une insurrection révolutionnaire, au moment du déclenchement de la guerre ont été maitrisées et les foules se sont résignées comme dans le reste du pays.

Pour ce qui est du clivage entre ville et campagne, il est possible d'établir une équation qui paraît valable dans toutes les recherches. Si les mouvements neutralistes sont idéologiquement enracinés dans les villes, ils sont souvent en position minoritaire par rapport aux interventistes. Au contraire, c'est à la campagne que le sentiment pacifiste était le plus répandu, mais les chercheurs ont démontré que les protestations dans ces zones n'ont pas de rapport avec les mouvements idéologisés. À ce propos, le cas de la Sardaigne est remarquable car en 1914, il y avait déjà des émeutes dans la région qui versait dans une crise économique qui avait entraîné la population dans une terrible famine, que ni les gouvernements locaux ni le gouvernement central n'avaient les moyens de résoudre.

Le jugement est tranchant : si les mouvements neutralistes avaient su canaliser les besoins des masses dans les campagnes et s'ils avaient réussi à les mobiliser ils auraient peut-être pu changer la donne. L'ouvrage, bien que loin d'être exhaustif, est passionnant. Il démontre non seulement que l'on peut interroger les archives locales pour avoir des réponses à des questions nationales, mais également que ces dernières sont bien loin d'être épuisées.

\section{Eleonora Lega}

EA $4574 \mathrm{SPH}$

Université Bordeaux Montaigne

Université de L'Insubrie, Varèse eleonoralega@gmail.com 